\title{
Dynamical Effects on the Geometric Phase
}

\author{
Laura Meißner $^{1}$, Tore Niermann ${ }^{1}$, Dirk Berger ${ }^{2}$ and Michael Lehmann ${ }^{1}$ \\ 1. Technische Universität Berlin, Institut für Optik und Atomare Physik, Berlin, Germany. \\ 2. Technische Universität Berlin, Zentraleinrichtung für Elektronenmikroskopie, Berlin, Germany.
}

Knowledge about the three dimensional strain field of semiconductor heterostructures improves the understanding and growth of semiconductor devices like light emitting diodes. Large-scale strain fields as present in such structures can be measured by means of dark-field electron holography or by related methods like geometric phase analysis. These methods allow recording the geometric phase of a reflected beam. This geometric phase is proportional to the displacement in the specimen, when the displacement is uniform along the beam direction.

We investigate the influence of dynamical scattering on these geometric phases in presence of 3D displacement fields, i.e. displacement-fields that also vary in the beam direction. An understanding of this interplay of dynamical scattering and the 3D strain fields is necessary for correct interpretation of the geometric phase.

The investigated samples contain a pseudomorphically grown InGaAs-layer with embedded InGaAsquantum dots within a GaAs-matrix. Utilizing a focused ion beam we prepared a lamella, which contains this InGaAs layer under an angle of $45^{\circ}$ [1]. An inclined layer allows correlating the position of the associated displacements in depth of the specimen with their lateral position (Figure 1). In calculations we approximated the quantum dots by spherical precipitates and added the displacement fields of the precipitates and the displacement field of the InGaAs-layer.

To visualize the influence of dynamic scattering on the geometric phase we performed calculations based on the numerical propagation of the Darwin-Howie-Whelan equations [2]. Figure 2 shows the phases of the (400)-beam, which lays within the growth plane and thus is only sensitive to displacements caused by the quantum dots, and not sensitive to the displacement of the layer in the [001] growth direction. A periodic change of the geometric phases of the quantum dots is apparent: the phases of the two central dots exhibit the opposite sign compared to the outer quantum dots, while the displacement field around all dots is the same.

Theoretically, these dynamic effects can be described by means of a weighting function [3, 4]. Figure 3 shows this weighing function for different sample thicknesses and depths. The characteristic sign change for the quantum dots in depths of 50 and $75 \mathrm{~nm}$ (as observed in Figure 2) can also be found in this function for a $125 \mathrm{~nm}$ thick sample.

Figure 4 shows the reconstructed amplitudes and phases of experimentally obtained bright-field and ( 0 22) dark-field holograms of this structure. These holograms are recorded with the (0-22) reflection strongly excited. The (0-22) beam is sensitive to the layer displacement in growth direction. Due to the different depths of this displacement the oscillating behavior can be directly observed in the phase. Here, the displacements caused by the quantum dots itself are not observable anymore, since in comparison the effects of the InGaAs layer itself are much stronger. [5] 
References:

[1] D. Berger, S. Selve, U.U.R. Hömpler, L. Meißner, MC 2017 Proceedings, p. 479.

[2] M. de Graef, "Introduction to Conventional Transmission Electron Microscopy", Cambridge University Press, Cambridge, (2003).

[3] E. Javon et al, Ultramicroscopy 147 (2014), p. 70.

[4] A. Lubk et al, Ultramicroscopy 136 (2014), p. 42.

[5] The authors acknowledge financial support from the Deutsche Forschungsgemeinschaft (SFB 787).

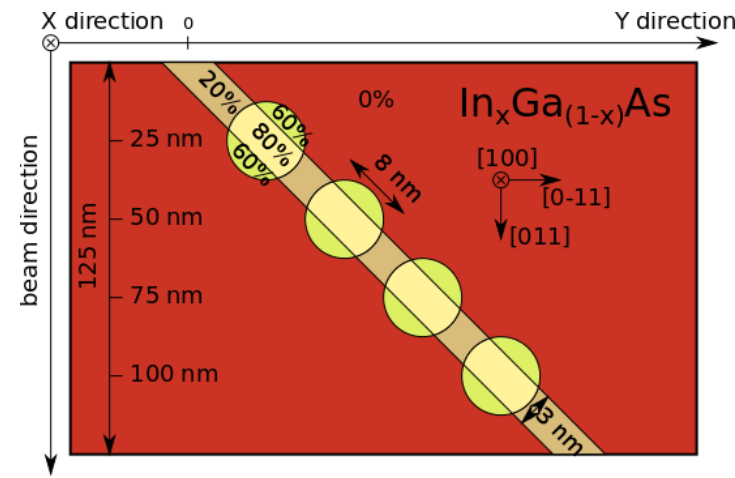

Figure 1. Due to the $45^{\circ}$ inclination of the layer, the y-position of a quantum dot also corresponds to a specific depth within the specimen.

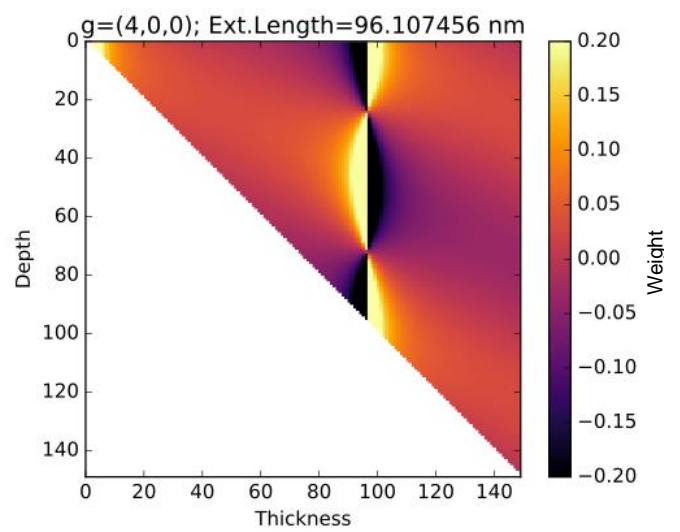

Figure 3. The weighting function for different depths and thicknesses for (400) strong beam conditions.

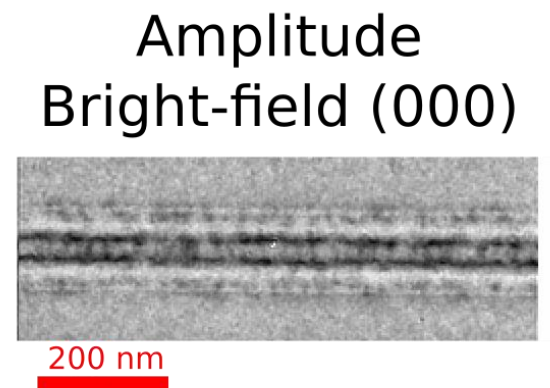

\section{Amplitude} Bright-field (000)
Amplitude
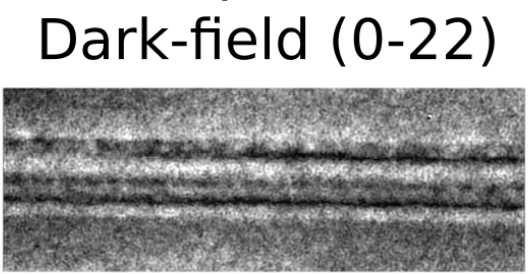

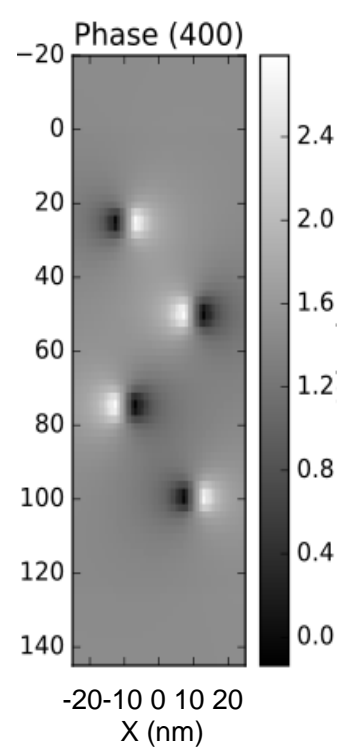

Figure 2. Calculated dark field phase of the (400)beam under (400) strong beam conditions.

Figure 4. Measured amplitudes and phases by means of bright-field and dark-field electron holography under (0-22) strong beam conditions. 